\title{
26417 - CEREBRAL OXYGEN SATURATION AND LEFT VENTRICULAR FUNCTION
}

\section{Catherine Paquet MD, Pierre Couture, MD FRCPC; Michel Carrier, MD FRCSC; Jean-Claude Tardif, André Y Denault, MD, FRCPC; Montreal Heart Institute, Montreal, QUEBEC, Canada}

INTRODUCTION: Near-infrared spectroscopy (NIRS) is a non-invasive and continuous monitor to measure the balance between cerebral oxygen delivery and consumption. So far no study has evaluated and compared the interaction between regional oxygen saturation (rSO2) values and cardiac function measured using both transesophageal echocardiography and the pulmonary artery catheter. We hypothesized that the evaluation of cardiac function with the transesophageal echocardiography (TEE) will correlate better with $\mathrm{rSO} 2$ values than through the measurements of cardiac performance obtained via a pulmonary artery catheter.

METHODS: With approval of our Ethic and Research Committee, a retrospective analysis of a TEE database included consecutive patients undergoing various cardiac surgery between June 2002 and November 2004. Patients were selected if in addition to the TEE exam, cerebral oxygen saturation measured by NIRS during their anesthesia and a pulmonary artery catheter was used. Demographic data, preoperative medications and Parsonnet score, the nature of the surgery and preoperative comorbidities were collected. The TEE exam included the measurement of fractional area change (FAC) and the regional wall motion score index (RWMSI). Left ventricular diastolic function was evaluated according to published guidelines. Cardiac output (CO) was measured with thermodilution and stroke volume (SV) calculated by dividing the SV by the heart rate. Cardiac index (CI) and stroke volume index (SVI) obtained by dividing CO and SV by the body surface area. The echocardiographic exam and the hemodynamic profile were obtained after the induction of anesthesia and compared to the baseline NIRS value. Ttest and chi-square were used to compare data according to their distribution. $\mathrm{P}<0.05$ was considered significant.

RESULTS: A total of 99 patients was included in the analysis. There were 65 men and 34 women. The mean age was $65 \pm 12.6$ years old, Parsonnet score $18.0 \pm 12.7$ and mean cerebral oxygen saturation values were $65.7 \pm 11.2$ and $64.3 \pm 11.1$, respectively on the left and right size. The mean FAC was $44.7 \pm 14.4 \%$, RWMSI $1.3 \pm 0.5$, cardiac index (CI) $1.9 \pm 0.4 \mathrm{~L}$ min- $1 \mathrm{~m}-2$ and stroke index (SI) $36.1 \pm 7.9 \mathrm{ml}$ beat $\mathrm{m}-2$. There was a significant positive correlation between left and right $\mathrm{rSO} 2$ values and the $\mathrm{FAC}(\mathrm{p}<0.001$ and $\mathrm{p}=0.0021)$, the RWMSI ( $\mathrm{p}=0.0011$ and $\mathrm{p}=0.0156$ ), left ventricular diastolic dysfunction $(\mathrm{p}=0.0076$ and $\mathrm{p}=0.003$ ) but no correlation with CI and SI was observed. DISCUSSION: Our results indicate a better correlation between cerebral oxygen saturation values and the cardiac function evaluated by TEE compared to the hemodynamic values. This could be explained by an increase in cerebral oxygen extraction in patients with reduced cardiac function. 